\title{
High-resolution photoluminescence spectroscopy of Sn-doped ZnO single crystals
}

\author{
E. Senthil Kumar ${ }^{1}$, F. Mohammadbeigi ${ }^{1}$, L. A. Boatner ${ }^{2}$ and S. P. Watkins ${ }^{1}$ \\ ${ }^{1}$ Department of Physics, Simon Fraser University, Burnaby, BC, Canada-V5A1S6 \\ ${ }^{2}$ Materials Science and Technology Division, Oak Ridge National Laboratory, Oak Ridge, \\ Tennessee 37831, USA \\ E-mail: simonw@sfu.ca
}

\begin{abstract}
.
Group IV donors in $\mathrm{ZnO}$ are poorly understood, despite evidence that they are effective n-type dopants. Here we present high-resolution photoluminescence (PL) spectroscopy studies of unintentionally doped and $\mathrm{Sn}$-doped $\mathrm{ZnO}$ single crystals grown by the chemical vapor transport method. Doped samples showed greatly increased emission from the $\mathrm{I}_{10}$ bound exciton transition that was recently proven to be related to the incorporation of $\mathrm{Sn}$ impurities based on radioisotope studies. The PL linewidths are exceptionally sharp for these samples, enabling a clear identification of several donor species. Temperature-dependent PL measurements of the $\mathrm{I}_{10}$ line emission energy and intensity dependence reveal a behavior that is similar to other shallow donors in $\mathrm{ZnO}$. Ionized donor bound-exciton and two-electron satellite transitions of the $\mathrm{I}_{10}$ transition are unambiguously identified and yield a donor binding energy of $71 \mathrm{meV}$. In contrast to recent reports of Ge-related donors in $\mathrm{ZnO}$, the spectroscopic binding energy for the $\mathrm{Sn}$ related donor bound exciton follows a linear relationship with donor binding energy (Haynes rule) similar to recently observed carbon related donors, and confirming the shallow nature of this defect center, which was recently attributed to a $\mathrm{Sn}_{\mathrm{Zn}}$ double donor compensated by an unknown single acceptor.
\end{abstract}


Keywords: zinc oxide, photoluminescence, electrical transport, dopants, $\mathrm{Sn}$

\section{INTRODUCTION}

The electronic properties of $\mathrm{ZnO}$ have been extensively studied by means of low-temperature photoluminescence (PL) spectroscopy.[1-5] Bound exciton transitions associated with group III donor impurities such as $\mathrm{Al}, \mathrm{Ga}$ and In were unambiguously identified with controlled intentional doping experiments.[1-7] These characteristic transitions were previously denoted as I-lines and identified as arising from neutral $\left(\mathrm{D}^{0} \mathrm{X}\right)$ and ionized $\left(\mathrm{D}^{+} \mathrm{X}\right)$ donor bound-exciton transitions. $\mathrm{D}^{0} \mathrm{X}\left(\mathrm{I}_{6}, \mathrm{I}_{8}\right.$, and $\left.\mathrm{I}_{9}\right)$ and $\mathrm{D}^{+} \mathrm{X}\left(\mathrm{I}_{0}, \mathrm{I}_{1}\right.$ and $\left.\mathrm{I}_{2}\right)$ of the substitutional group III donors $\mathrm{Al}$, $\mathrm{Ga}$ and In donors are now well established.[1] Shallow hydrogen and antimony related $\mathrm{D}^{0} \mathrm{X}$ transitions have also been observed. Unlike the group III donors, these do not have $\mathrm{D}^{+} \mathrm{X}$ transitions $[1,2,4]$ due to their very small donor binding energies. The chemical nature of several of the I-lines in the near-band-edge region for example, $\mathrm{I}_{7}, \mathrm{I}_{5}, \mathrm{I}_{3}$, is still unknown.

When compared with group-III impurities, the electronic nature of the group-IV impurities (C, $\mathrm{Si}, \mathrm{Ge}$ and $\mathrm{Sn})$ in $\mathrm{ZnO}$ is not clearly understood. Group-IV impurities are potential double donors in $\mathrm{ZnO}$ when substituting for $\mathrm{Zn}$ sites.[8, 9] Cullen et al. have reported an unusual Ge-related donor bound exciton transition at $3324 \mathrm{meV}$ in bulk $\mathrm{ZnO}$ crystals which binds excitons much more deeply than expected based on its shallow final state donor binding energy.[10] Recently, Mohammadbeigi et al. have observed carbon-related shallow donor $\mathrm{D}^{0} \mathrm{X}$ transitions, labeled Z-lines, in carbon-doped $\mathrm{ZnO}$ nanowires grown by metalorganic vapor phase epitaxy.[11] Pulsed laser deposited (PLD) Si-doped $\mathrm{ZnO}$ thin films exhibited a two-orders-ofmagnitude increase in conductivity compared with un-doped samples.[9] Sn-doped $\mathrm{ZnO}$ thin films exhibited increased conductivity only at low Sn concentrations (0.1 at. \%) and showed a 
larger resistivity at higher Sn concentrations (1-2 at \%).[12] Recent PL measurements on radioactive ${ }^{117} \mathrm{Ag}$ implanted $\mathrm{ZnO}$ single crystals showed an appearance of the $\mathrm{I}_{10}$-line at 3353 meV.[13] The center responsible for the $\mathrm{I}_{10}$ line was conclusively shown to contain $\mathrm{Sn}$, based on the implantation of radioactive ${ }^{117} \mathrm{Ag}$ which eventually decays into stable ${ }^{117} \mathrm{Sn}$. Meyer et al. have observed the $\mathrm{I}_{10}$ line in Li- and Na-doped $\mathrm{ZnO}$ single crystals [1] and previously suggested that the $\mathrm{I}_{10}$ center contains $\mathrm{Li}$ or $\mathrm{Na}$, however this remains to be proven.

In this study, we performed high-resolution PL experiments on $\mathrm{ZnO}$ single crystals intentionally doped with $\mathrm{Sn}$ impurities. We show that the intentionally Sn-doped samples have a consistently higher PL intensity of the $\mathrm{I}_{10}$ line and the related $\mathrm{I}_{10}{ }^{+}\left(\mathrm{D}^{+} \mathrm{X}\right)$ transition compared with un-doped samples. We demonstrate that the $\mathrm{I}_{10}$ defect shares many similarities with the group III donors in terms of its bound-exciton PL properties - such as thermal activation and peak energy shift with temperature, and that this is consistent with a shallow donor impurity binding a single electron. We confirm the two electron satellite (TES) transitions and $\mathrm{D}^{+} \mathrm{X}$ transitions associated with $\mathrm{Sn}$ impurities. We observe that $\mathrm{I}_{10}$ follows the empirical relationship between localization energy and binding energy often referred to as Hayne's rule, and that it follows the same trend as the common group III and other shallow donors, indicating a similar relatively shallow central cell potential.

\section{EXPERIMENTAL}

Unintentionally doped and $\mathrm{Sn}$-doped $\mathrm{ZnO}$ single crystals were grown using the chemical vapor transport (CVT) method. The growth of single crystals of $\mathrm{ZnO}$ from the vapor phase is accomplished by the reduction of polycrystalline spheres of $\mathrm{ZnO}$ that are placed in an alumina tube (Fig. 1). The process consists of two steps: the initial step involves the reduction of $\mathrm{ZnO}$ with hydrogen gas to provide a concentration of zinc in the region where the bulk crystal growth 
is to take place. This is achieved through the reaction: $\mathrm{ZnO}(\mathrm{s})+\mathrm{H}_{2}(\mathrm{~g}) \rightarrow \mathrm{Zn}(\mathrm{g})+\mathrm{H}_{2} \mathrm{O}(\mathrm{g})$. This reaction occurs in a hot zone $\left(1250^{\circ} \mathrm{C}\right)$ of the tube furnace. The zinc vapor is transported to a cooler growth region by a nitrogen carrier gas where the crystals grow by means of the reaction: $\mathrm{Zn}(\mathrm{g})+(1 / 2) \mathrm{O}_{2}(\mathrm{~g}) \rightarrow \mathrm{ZnO}(\mathrm{s})$, which is accompanied by the reaction: $\mathrm{H}_{2}(\mathrm{~g})+(1 / 2) \mathrm{O}_{2}(\mathrm{~g}) \rightarrow$ $\mathrm{H}_{2} \mathrm{O}(\mathrm{g})$. $\mathrm{ZnO}$ crystals with dimensions of several centimeters can be grown by this technique. Sn-doping was achieved by mixing $0.15 \mathrm{~mol} \%$ of $\mathrm{SnO}_{2}$ with the starting $\mathrm{ZnO}$ polycrystalline material. Variations in Sn doping observed by $\mathrm{PL}$ are not attributed to changes in the $\mathrm{SnO}_{2}$ precursor concentration, but rather to variations in the incorporation of Sn in different parts of the growth chamber due to variations in the temperature and other local growth conditions. The CVT process yielded needle shaped crystal ranging in diameter from $\sim 0.2 \mathrm{~mm}$ to $2 \mathrm{~mm}$ in diameter and up to $15 \mathrm{~mm}$ in length. The larger diameter samples were used for electrical resistivity measurements. High-resolution PL measurements were carried out at $4.2 \mathrm{~K}$, in a liquid helium cryostat, by exciting the samples with the $325.031 \mathrm{~nm}$ line of a $\mathrm{HeCd}$ laser with a total power of $25 \mathrm{~mW}$. The luminescence was dispersed by a double monochromator with a $0.85 \mathrm{~m}$ focal length and with a spectral resolution of $0.08 \mathrm{meV}$. A Hamamatsu R585 photomultiplier tube was used to count the photons. The wavelengths were corrected using the refractive index of air and converted to energy using a conversion factor of $1239841.7 \mathrm{eV} \mathrm{nm}$. Indium was used for electrical contracts.

\section{RESULTS AND DISCUSSION}

Figure 1 shows the $4.2 \mathrm{~K}$ PL spectra of the unintentionally doped (A) and Sn doped $\mathrm{ZnO}$ single crystals (B,C,D,E). The PL spectrum of the unintentionally doped $\mathrm{ZnO}$ is mainly dominated by the $\mathrm{D}^{0} \mathrm{X}$ transitions $\mathrm{I}_{6}, \mathrm{I}_{8}$ and $\mathrm{I}_{9}$ corresponding to residual $\mathrm{Al}, \mathrm{Ga}$ and In donors. Following the convention of Allen et al. [14] their corresponding $\mathrm{D}^{+} \mathrm{X}$ transitions are denoted as 
$\mathrm{I}_{6}{ }^{+}(\mathrm{Al}), \mathrm{I}_{8}{ }^{+}(\mathrm{Ga})$ and $\mathrm{I}_{9}^{+}(\mathrm{In})$, which were previously labeled $\mathrm{I}_{0}(\mathrm{Al}), \mathrm{I}_{1}(\mathrm{Ga})$ and $\mathrm{I}_{2}(\mathrm{In})$ respectively.[1] The line positions of these transitions are in agreement with previous reports on bulk $\mathrm{ZnO}$ crystals and intentionally doped nanowires.[1, 4, 5] Unintentionally doped crystals also showed another transition with relatively weak PL intensity at $3353.2 \mathrm{meV}$, which is very close to the previously assigned $\mathrm{I}_{10}$-line position $(3353.1 \mathrm{meV})$.[1] These transitions are very sharp, with linewidths as low as $54 \mu \mathrm{eV}\left(\mathrm{I}_{10}\right), 170 \mu \mathrm{eV}\left(\mathrm{I}_{9}\right)$ and $260 \mu \mathrm{eV}\left(\mathrm{I}_{8}\right)$ revealing the low concentrations (estimated $\leq 10^{17} \mathrm{~cm}^{3}$ ) of residual shallow impurities in these crystals. The samples with significant $\mathrm{I}_{10}$ doping tend to have a feature at 3363.2 meV labeled $\mathrm{I}_{10}{ }^{+}$in Fig. 1 , which was previously attributed to the $\mathrm{D}^{+} \mathrm{X}$ transition of $\mathrm{I}_{10}[15]$ however in that work the chemical origin of $I_{10}$ was not yet known.

The PL spectra (B-E) in Fig.1 correspond to Sn-doped ZnO crystals; however the actual Sn concentration is not known but it appears to be very low based on the sharpness of the observed PL lines. The unintentionally doped $\mathrm{ZnO}$ crystals (e.g. A in Fig. 1) are highly transparent, with no apparent color. The doped samples exhibit varying amounts of blue color ranging from sample to sample within the same growth run. As a trend, the darker blue samples exhibit a high relative intensity of $I_{10}$ compared with the other residual donors such as $I_{9}$. Also, the linewidth of the $\mathrm{I}_{10}$ line increases from $54 \mu \mathrm{eV}$ for the un-doped sample to $170 \mu \mathrm{eV}$ for the darkest blue samples. These samples also showed strong emission at $3333.3 \mathrm{meV}$ corresponding to the socalled Y-line (not shown) which has been attributed to excitons bound deeply to donor centers localized at structural defects.[1]

Meyer et al. have reported the $\mathrm{I}_{10}$ line in both $\mathrm{Li}$ - and Na-doped $\mathrm{ZnO}$ crystals [1]. Lidoped crystals showed a higher integrated PL intensity for the $\mathrm{I}_{10}$ line compared with Na-doped crystals.[1] However, PL measurements on systematically Li-doped $\mathrm{ZnO}$ samples were unable to 
unambiguously assign $\mathrm{Li}$ to the $\mathrm{I}_{10}$ line. Cullen et al. have also performed PL measurements on $\mathrm{ZnO}$ crystals implanted with the radioactive impurity ${ }^{117} \mathrm{Ag}$, which decays into radioactive ${ }^{117} \mathrm{In}$, and then to stable ${ }^{117} \mathrm{Sn}$, resulting in the appearance of the $\mathrm{I}_{10}$-line, that was absent in virgin samples.[13] The time evolution of the $\mathrm{I}_{10}$ emission occurred with a rate consistent with the known decay of ${ }^{117} \mathrm{In}$ to ${ }^{117} \mathrm{Sn}$ - thus strongly arguing that $\mathrm{Sn}$ is present on a $\mathrm{Zn}$ lattice site as the $\mathrm{I}_{10}$ defect. In order to explain the observed single donor charge state they attributed the $\mathrm{I}_{10}$-line to the donor complex, $\mathrm{Sn}_{\mathrm{Zn}}-\mathrm{Li}$ Zn, where Li acts as a compensating acceptor.[13] Recently, Rakshit et al. have observed a transition at $3355 \mathrm{meV}$, which is close to the $\mathrm{I}_{10}$-line in Sn-doped $\mathrm{ZnO}$ nanostructures grown by the vapor-solid method.[16]

PL measurements as a function of temperature indicate whether an exciton binding center has a shallow or deep nature based on the energy shift of the transitions [17] and also whether the transition is associated with a ground state or excited state - based on the thermalization behavior.[18] Fig. 2 (a) shows the energy shift of the $I_{8}, I_{9}, I_{10}$ and $Y$ lines as a function of temperature in the range 4.2-45 K. The Y-lines are associated with donor bound excitons with deeply bound excitons [18] in other words, the exciton binding energy is much larger than would be predicted based on the shallow donor final state. This will be discussed in detail later in the paper. All the data are fitted using Eqn. 1 given by Viña et al. based on a Bose-Einstein model [19],

$$
E(T)=E_{0}-\frac{\alpha}{\exp \left(\frac{\Theta}{T}\right)-1}
$$

where, $E_{0}$ is the emission energy at $\mathrm{T}=0 \mathrm{~K}, \alpha$ is a parameter associated with electron-phonon coupling, and $\Theta$ is the effective phonon temperature. The solid lines represent the least squares 
fits to the data points. The best fit yielded the fitting parameter $\alpha=0.11 \pm 0.01 \mathrm{eV} / \mathrm{K}$ for $\mathrm{I}_{8}, \mathrm{I}_{9}$ and $\mathrm{I}_{10}$ and $0.31 \pm 0.05 \mathrm{eV} / \mathrm{K}$ for the $\mathrm{Y}$ line. The other fitting parameter $\theta$ for the $\mathrm{I}_{8}, \mathrm{I}_{9}, \mathrm{I}_{10}$ and $\mathrm{Y}$ lines is $96 \pm 5 \mathrm{~K}, 86 \pm 8 \mathrm{~K}, 97 \pm 6 \mathrm{~K}$ and $82 \pm 7 \mathrm{~K}$ respectively. The amount of energy shift $(\Delta E)$ for the $\mathrm{I}_{10}$ line, in the temperature range from $4.2 \mathrm{~K}$ to $45 \mathrm{~K}$, is exactly the same as that of the group III impurities, $\mathrm{I}_{8}$ and $\mathrm{I}_{9}$. The energy shift of the $\mathrm{Y}$-line is 5 times larger than that of the $\mathrm{I}_{8}$, $\mathrm{I}_{9}$ and $\mathrm{I}_{10}$ lines over the same temperature range. This confirms that the impurity responsible for the $\mathrm{I}_{10}$ line is a shallow donor defect with a spatial variation of the central cell potential similar to the group III donors.

The thermalization behavior of the $\mathrm{I}_{10}$ line [Fig. 2(b)] is also very similar to other shallow neutral donor bound-exciton transitions. The intensity of the $\mathrm{I}_{10}$ line falls steadily with increasing temperature from $4.2 \mathrm{~K}$ to $25 \mathrm{~K}$ [Fig. 2(c)] and then drops rapidly with a further increase in temperature. This is a typical characteristic of a ground-state transition and is similar to the behavior of the $I_{9}$ and other shallow lines. The activation energies of the $I_{10}$ and $I_{9}$ transitions are obtained by fitting [Fig. 2c)] using a quasi-empirical relation [20],

$$
I=\frac{I_{0}}{1+C_{1} \exp \left(\frac{-E_{a 1}}{k T}\right)+C_{2} \exp \left(\frac{-E_{a 2}}{k T}\right)}
$$

where $E_{a 1}$ and $E_{a 2}$ are activation energies and $C_{1}$ and $C_{2}$ are degeneracy ratios. Fitting the data points using the model in Eqn. 2 yielded activation energies $E_{a 2}$ of $19 \pm 2 \mathrm{meV}$ and $22 \pm 2 \mathrm{meV}$ for $\mathrm{I}_{9}$ and $\mathrm{I}_{10}$ respectively. These values are close to the respective spectroscopic binding energies of the $\mathrm{I}_{9}$ and $\mathrm{I}_{10}$ transitions of 18.8 and $22.4 \mathrm{meV}$. This again confirms that the $\mathrm{I}_{10}$ line is associated with shallow impurity centers. In contrast, donor bound exciton transitions with deeply bound excitons such as the Y-line[1] or the Ge-related $\mathrm{D}^{0} \mathrm{X}$ observed by Johnston et al.[6] 
typically thermalize very rapidly and show a large discrepancy between the activation energy and spectroscopic binding energy. This discrepancy has been attributed to the possibility that thermal dissociation involves the removal of a single particle rather than the exciton as a whole, leaving the other particle bound in a more localized state.[17]

The $\mathrm{I}_{10}$ line is known to be due to $\mathrm{D}^{0} \mathrm{X}$ emission based on the observation of two-electron satellite (TES) transitions, which are $\mathrm{D}^{0} \mathrm{X}$ transitions where the final state electron is left in an excited state. We observe a two-electron satellite transition at $3294.7 \mathrm{meV}$ as shown in Fig. 3, which is close to the previously reported $2 p$ transition of the $I_{10}$ line. [1] Locating the $2 p$ level allows us to estimate the energy difference between the $1 \mathrm{~s}$ and $2 p \mathrm{D}^{0} \mathrm{X}$ transitions. Based on these results, we determined a donor binding energy for the $\mathrm{I}_{10}$ donor of $71.1 \mathrm{meV}$, which compares reasonably well with the value of $72.6 \mathrm{meV}$ obtained earlier.[1] It should be noted that the PL linewidths of the current samples are considerably sharper than previous reports.

It is useful to consider how the $\mathrm{I}_{10}$ transitions fit with trends observed in other shallow donors in $\mathrm{ZnO}$. Haynes observed an empirical rule for shallow donors in various semiconductors which states that the donor exciton binding energy is linearly related to the donor binding energy.[21] This rule is well observed for the group III donors and other shallow donors including a hydrogen-related defect $\left(\mathrm{I}_{4}\right)$. Meyer et al. previously showed that $\mathrm{I}_{10}$ obeys Haynes rule, although the chemical origin of the transition was not known at that time. In Fig 4(a) we plot our data for the Sn-related donor on a plot of $\mathrm{D}^{0} \mathrm{X}$ and $\mathrm{D}^{+} \mathrm{X}$ binding energy vs. donor binding energy together with several shallow donors in $\mathrm{ZnO}$ - including the recently observed carbon-related $\mathrm{D}^{0} \mathrm{X}$ transitions [Fig. 4(a)]. The $\mathrm{I}_{10}$ transition clearly follows the trends observed for other shallow donors including the carbon related ones. Also shown are the $2 \mathrm{~s}-2 \mathrm{p}$ splittings 
for these donors indicating a similar systematic increase in central cell correction for the $2 \mathrm{~s}$ donor state which correlates linearly with increasing donor binding energy.

Note that In and Sn have the same core electron structure. The simplest defect model assumes that the Sn-related donor sits on a $\mathrm{Zn}$ site (as confirmed by Cullen et al.[13]) and that the excess charge of the double donor is compensated by a yet unknown single acceptor impurity for example $\mathrm{Li}$ or $\mathrm{Na} \cdot[1,13]$ The compensating defect is unlikely to be a $\mathrm{Zn}$ vacancy since this would be expected to result in a neutral defect, since the $\mathrm{Zn}$ vacancy is a double acceptor. Likewise, we can rule out an isolated $\mathrm{Sn}_{\mathrm{Zn}}$ double donor since it is not expected to have $2 \mathrm{~s} / 2 \mathrm{p}$ excited final states due to the expected pairing of the electron spins.

While the behavior of $I_{10}$ is very similar to the known shallow single donors, it is in strong contrast to another well studied group IV impurity Ge which has a donor bound exciton emission at $3324 \mathrm{meV}$ [6]. The Ge-related defect exhibits a final state donor binding energy similar to shallow donors like $\mathrm{I}_{6}, \mathrm{I}_{8}, \mathrm{I}_{9}$, and $\mathrm{I}_{10}$ - but a much deeper exciton localization energy, as evident from Fig.4(a). Similar behavior is observed for the Y-line defect at $3333 \mathrm{meV}$, suggesting a similar possible origin. A detailed microscopic model for these transitions is not yet available.

To investigate the electrical quality of these CVT grown samples we also performed temperature-dependent resistivity measurements of samples A, B, C and E (Fig. 5). No significant correlations were observed between samples grown with different relative PL intensities of $\mathrm{Sn}$ impurities. Even the samples that showed the strongest $\mathrm{I}_{10}$ emission had room temperature resistivities much higher than those grown by PLD in Ref. 12, indicating that the Sn doping level was much lower in our case. The estimated room-temperature (295 K) resistivities are $3.92(\mathrm{~A}), 0.86(\mathrm{~B}), 19.7$ (C) and $2.35 \mathrm{Ohm} \cdot \mathrm{cm}$ (E). The sample E, which exhibited a 
relatively strong $\mathrm{I}_{10}$ line, showed evidence of impurity-band conduction at low temperature. The samples A, B, and C exhibited exponential freezeout over several orders of magnitude consistent with a very low carrier concentration and very high crystal quality. The activation energies for freezeout were observed to be $56.8 \mathrm{meV}(\mathrm{A}), 50.8 \mathrm{meV}(\mathrm{B})$ and $57.2 \mathrm{meV}(\mathrm{C})$. These values are far from the donor binding energy of Sn impurities of $71 \mathrm{meV}$ determined from twoelectron transitions in both this work and previous work.[1] This suggests that while the overall $\mathrm{I}_{10}$ defect concentration has increased, other shallow dopants dominate the electrical properties. At least for the doping levels investigated here, no significant effect on resistivity was observed that correlated with the intensity of the $\mathrm{I}_{10}$ emission.

\section{CONCLUSIONS}

We have investigated the optical and electrical properties of $\mathrm{ZnO}$ single crystals doped with Sn impurities. We find enhanced intensity of the $I_{10}$ transition in intentionally Sn-doped samples. We identify neutral and ionized donor bound exciton transitions of this defect, as well as the corresponding two-electron satellite transitions. The temperature and energy level dependence of the Sn-related $\mathrm{D}^{0} \mathrm{X}$ transitions show strong similarities with other shallow donors, including the recently observed carbon-related $\mathrm{D}^{0} \mathrm{X}$ emission, but they are in strong contrast to the behavior of Ge-related $\mathrm{D}^{0} \mathrm{X}$ complexes and the defect related Y-line luminescence centers in $\mathrm{ZnO}$. Our results add support to a previous defect model for $\mathrm{I}_{10}$ consisting of substitutional $\mathrm{Sn}_{\mathrm{Zn}}$ complexed with a single shallow acceptor.

\section{ACKNOWLEDGEMENTS:}

The support of the Natural Sciences and Engineering Research Council of Canada is gratefully acknowledged. Research at the Oak Ridge National Laboratory for one author (LAB) was supported by the U.S. Department of Energy, Office of Science, Basic Energy Sciences, 
Materials Sciences and Engineering Division.

\section{References}

[1] B.K. Meyer, H. Alves, D. Hofmann, W. Kriegseis, D. Forster, F. Bertram, J. Christen, A. Hoffmann, M. Straßburg, M. Dworzak, U. Haboeck, and A.V. Rodina Phys. Stat. Sol. (b) 241 (2004) 231.

[2] D.C Look, G.C. Farlow, P. Reunchan, S. Limpijumnong, S.B. Zhang, and K. Nordlund, Phys. Rev. Lett. 95 (2005) 225502.

[3] S.P. Watkins, Z.W. Deng, D.C. Li, and H. Huang, J. Appl. Phys. 110 (2011) 083506.

[4] E.S. Kumar, F. Mohammadbeigi, S. Alagha, Z.W. Deng, I.P. Anderson, T. Wintschel, and S.P. Watkins, Appl. Phys. Lett. 102, (2013) 132105.

[5] E.S. Kumar, I.P. Anderson, Z. Deng, F. Mohammadbeigi, T. Wintschel, D. Huang, and S.P. Watkins, Semicond. Sci. Technol. 28,( 2013) 045014.

[6] K. Johnston, M.O. Henry, D. McCabe, E. McGlynn, M. Dietrich, E. Alves, and M. Xia, Phys. Rev. B 73 (2006) 165212.

[7] S. Müller, D. Stichtenoth, M. Uhrmacher, H. Hofsäss, C. Ronning, and J. Röder Appl. Phys. Lett. 90 (2007) 012107.

[8] J.L. Lyons, A. Janotti, and C.G. Van de Walle, Phys. Rev. B 80 (2009) 205113.

[9] A.K. Das, P. Misra P, and L.M. Kukreja, J. Phys. D: Appl. Phys. 42 (2009)165405.

[10] J. Cullen, K. Johnston, E. McGlynn, M.O. Henry, D. Dunker, D.R. Yakovlev, and M. Bayer, Phys. Rev. B 87 (2013) 165202.

[11] F. Mohammadbeigi, E.S. Kumar, S. Alagha, I. Anderson, and S.P. Watkins, J. Appl. Phys. 116 (2014) 053516. 
[12] E. López-Ponce, J.L. Costa-Krämer, M.S. Martín-González, F. Briones, J.F. Fernández, A.C. Caballero, M. Villegas, and J. de Frutos, Phys. Stat. Sol (a) 203 (2006)1383.

[13] J. Cullen, D. Byrne, K. Johnston, E. McGlynn, and M.O. Henry Appl. Phys. Lett. 102 (2013) 192110.

[14] M.W. Allen, R. Heinhold, P. Miller, M.J.H. Henseler, R.J. Mendelsberg, S.M. Durbin, and R.J. Reeves, Appl. Phys. Lett. 103 (2013) 231109.

[15] B. K. Meyer, J. Sann, S. Lautenschläger, M. R. Wagner, and A. Hoffmann, Phys. Rev. B76 (2007) 184120.

[16] T. Rakshit, I. Manna, and S.K. Ray, AIP Advances 3, (2013) 112112.

[17] M.R. Wagner, G. Callsen, J.S. Reparaz, J.-H. Schulze, R. Kirste, M. Cobet, I.A. Ostapenko, S. Rodt, C. Nenstiel, M. Kaiser, A. Hoffmann, A.V. Rodina, M.R. Phillips, S. Lautenschläger, S. Eisermann, and B.K. Meyer, Phys. Rev. B 84 (2011) 035313.

[18] B.K. Meyer, J. Sann, S. Eisermann, S. Lautenschlaeger, M.R. Wagner, M. Kaiser, G. Callsen, J.S. Reparaz, and A. Hoffmann, Phys Rev B 82 (2010) 115207.

[19] L. Viña, S. Logothetidis, and M. Cardona, Phys. Rev. B 30 (1984) 1979.

[20] D. Bimberg, M. Sondergeld, and E. Grobe, Phys. Rev. B 4 ( 1971) 3451.

[21] J.R. Haynes, Phys. Rev. Lett. 4 (1960) 361. 


\section{Figure captions:}

Figure 1: 4.2 K PL spectra of the un-doped (A) and Sn-doped (B-E) CVT-grown ZnO single crystals.

Figure 2: (a) Temperature-dependent energy positions of the $\mathrm{I}_{8}, \mathrm{I}_{9}, \mathrm{I}_{10}$ and $\mathrm{Y}$ lines for a Sn-doped sample. Solid lines are fits to the data points using equation 2. (b) PL spectra of the Sn-doped sample at different temperatures from $4.2 \mathrm{~K}$ to $45 \mathrm{~K}$. (c) Temperature-dependent integrated PL intensity of $\mathrm{I}_{9}$ and $\mathrm{I}_{10}$ lines. The degeneracy ratios $C_{1}$ and $C_{2}$, which are assumed as independent fitting parameters in Eqn. 2 are 4 and 1403 for $\mathrm{I}_{9}$, and 6 and 4515 for the $\mathrm{I}_{10}$ line respectively.

Figure 3: Donor bound exciton and two-electron satellite spectra, corresponding to a Sn-doped sample.

Figure 4: (a) Localization energy for $\mathrm{D}^{0} \mathrm{X}$ and $\mathrm{D}^{+} \mathrm{X}$ versus donor binding energy for standard shallow donors $(\mathrm{H}, \mathrm{Al}, \mathrm{Ga}, \mathrm{In})$ as well as $\mathrm{I}_{10}$ and carbon-related donors (Z-lines) and "deeply bound" $\mathrm{D}^{0} \mathrm{X}$ transitions (Ge-related and Y line). (b) 2s-2p splitting for the various donors confirming the similarity of the 2 s central cell shift trend for the shallow donors, in contrast to the deeply bound $\mathrm{D}^{0} \mathrm{X}$ transitions (Ge-related and $\mathrm{Y}$-line).

Figure. 5: Resistivity vs. temperature data for the samples indicated in Fig. 1. 
FIGURES

FIG 1

Sn In $\mathrm{Ga}$ Al Sn In $\mathrm{Ga}$ Al

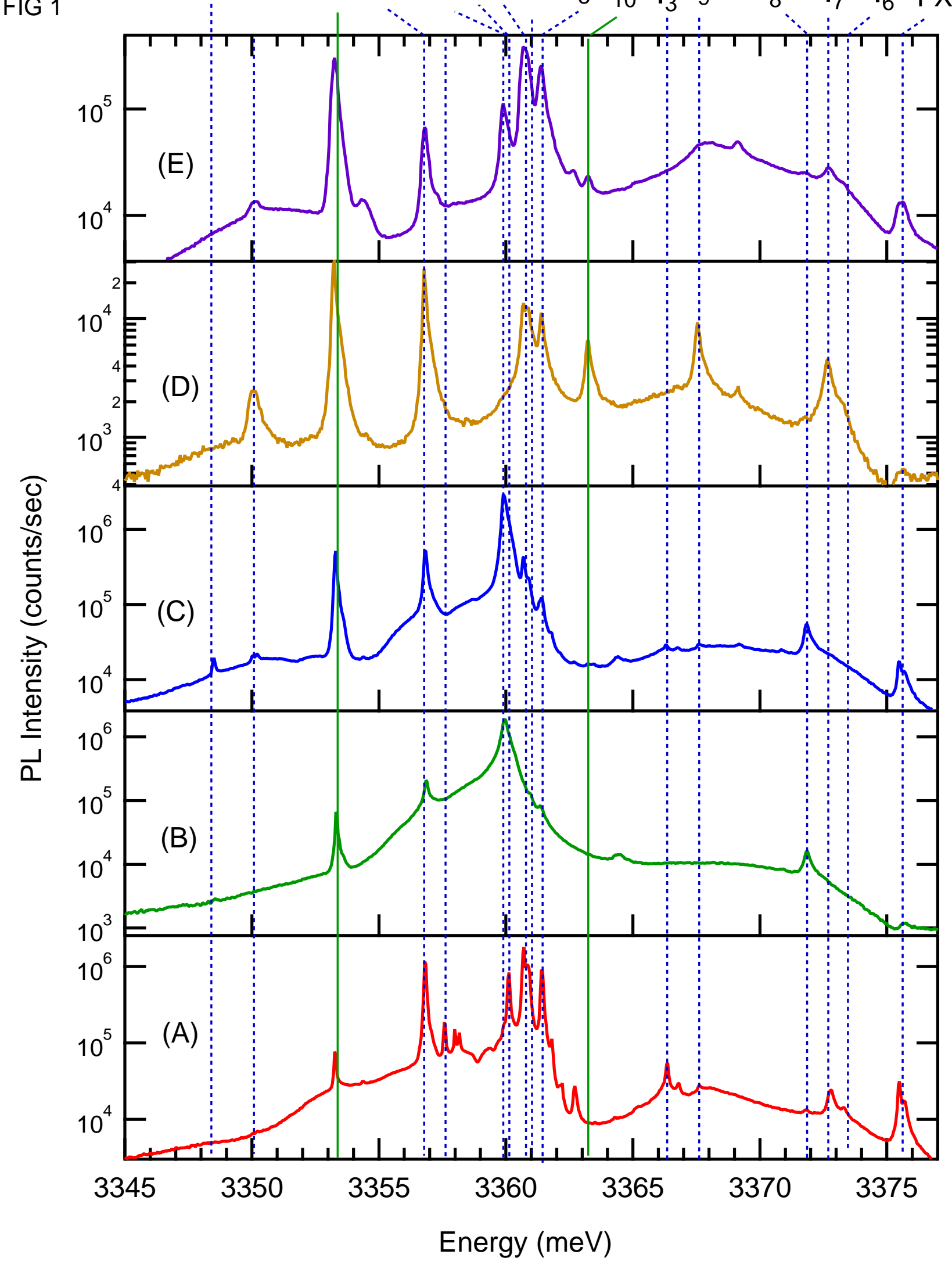



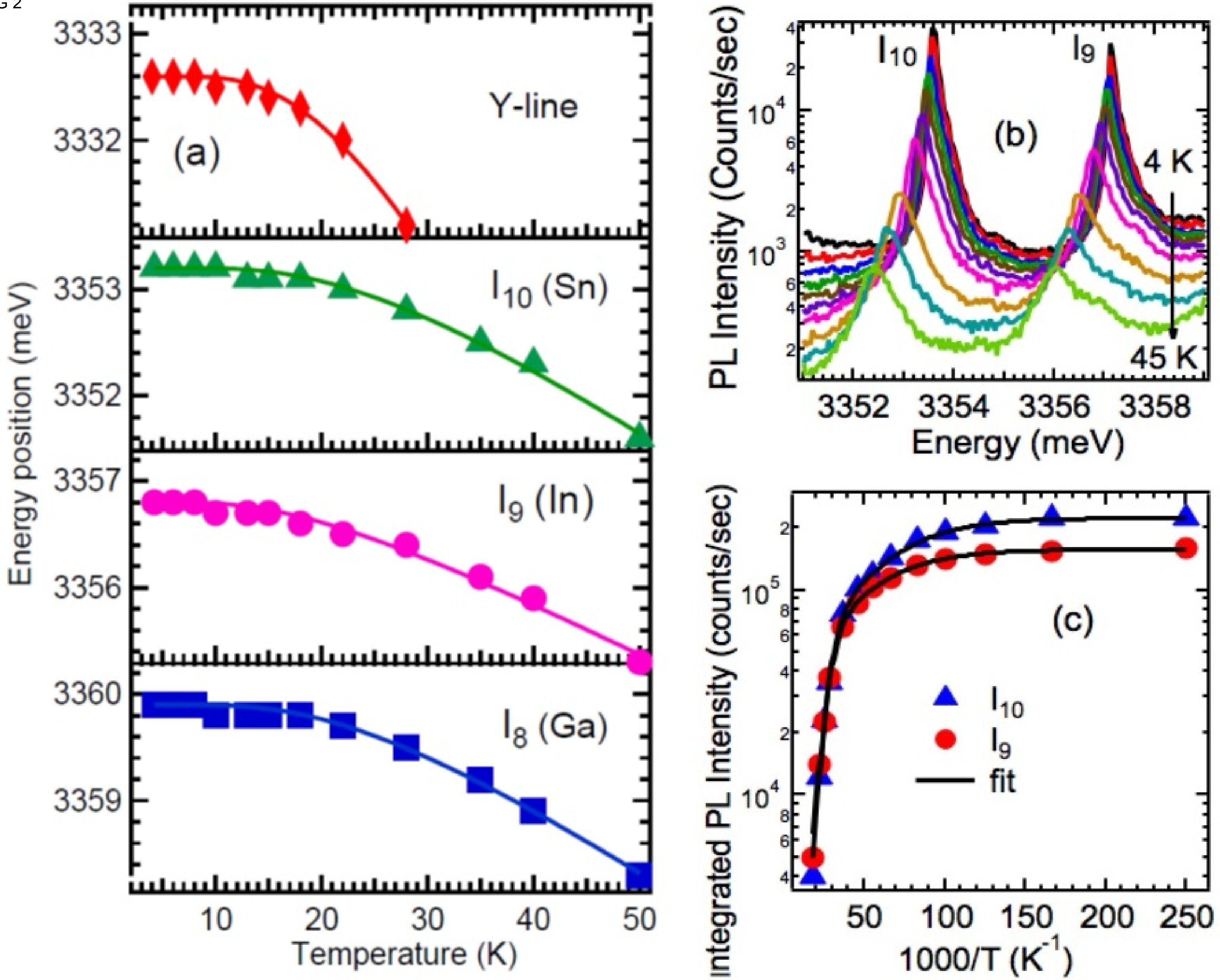


\section{FIG 3}

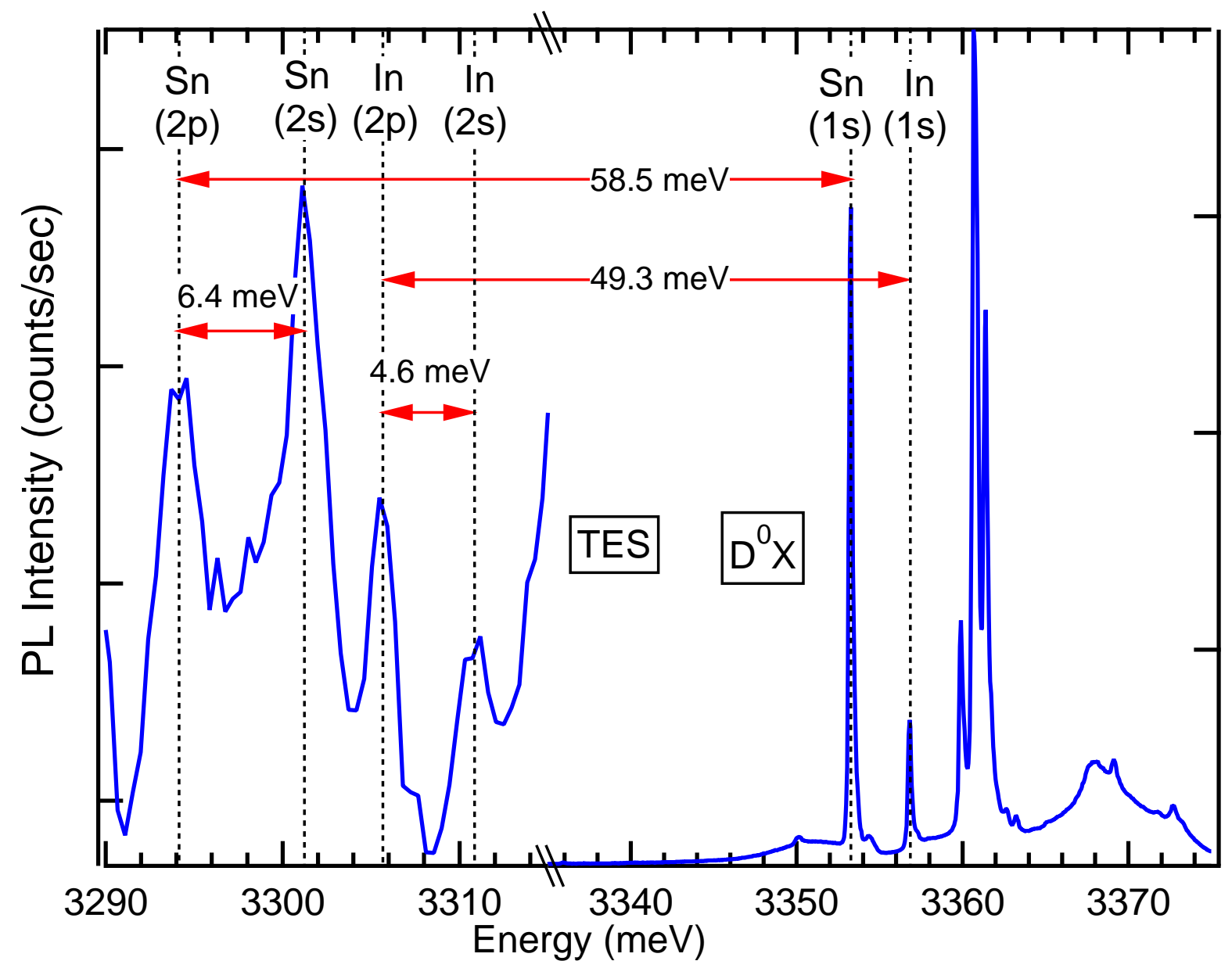



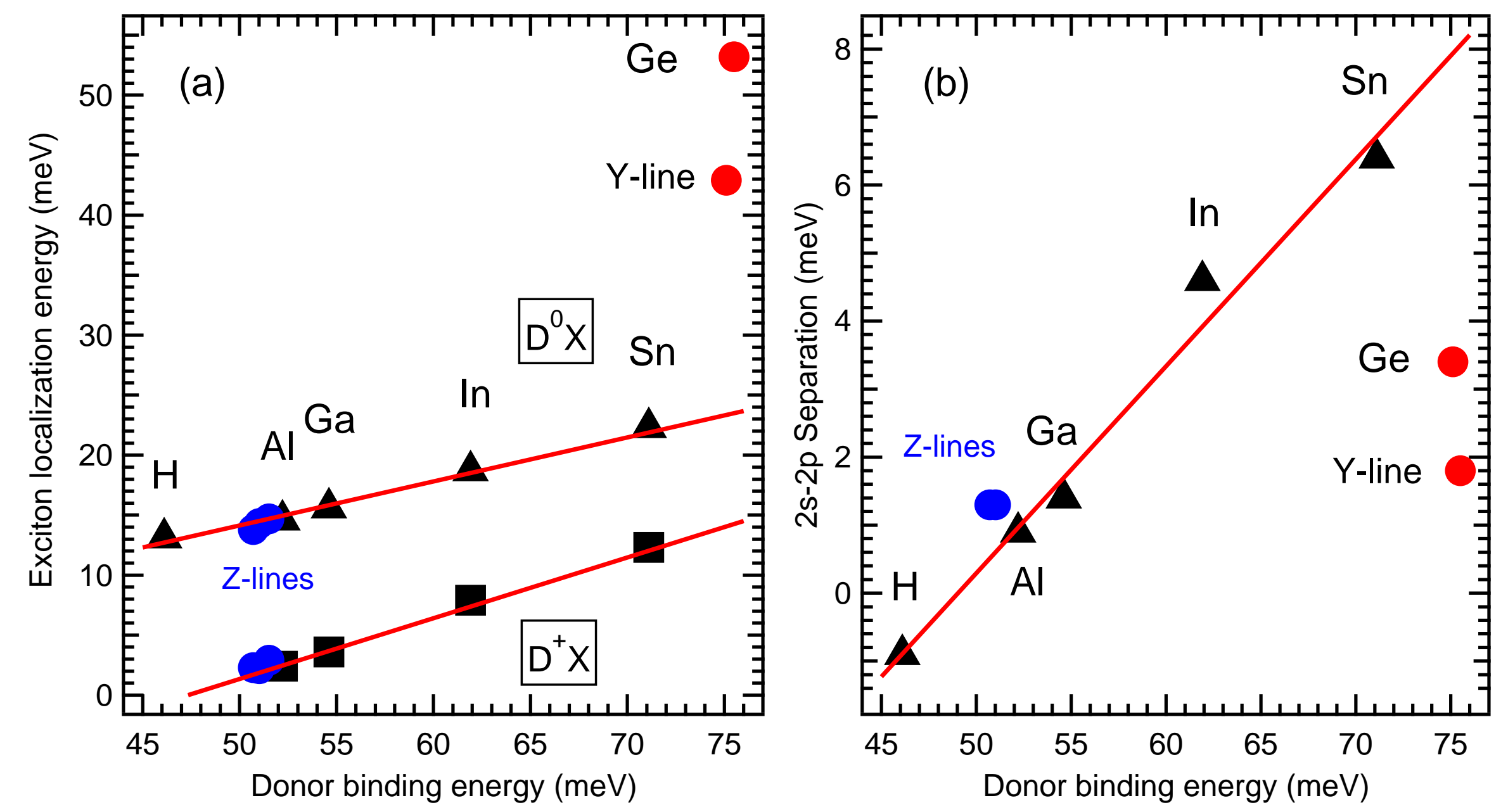


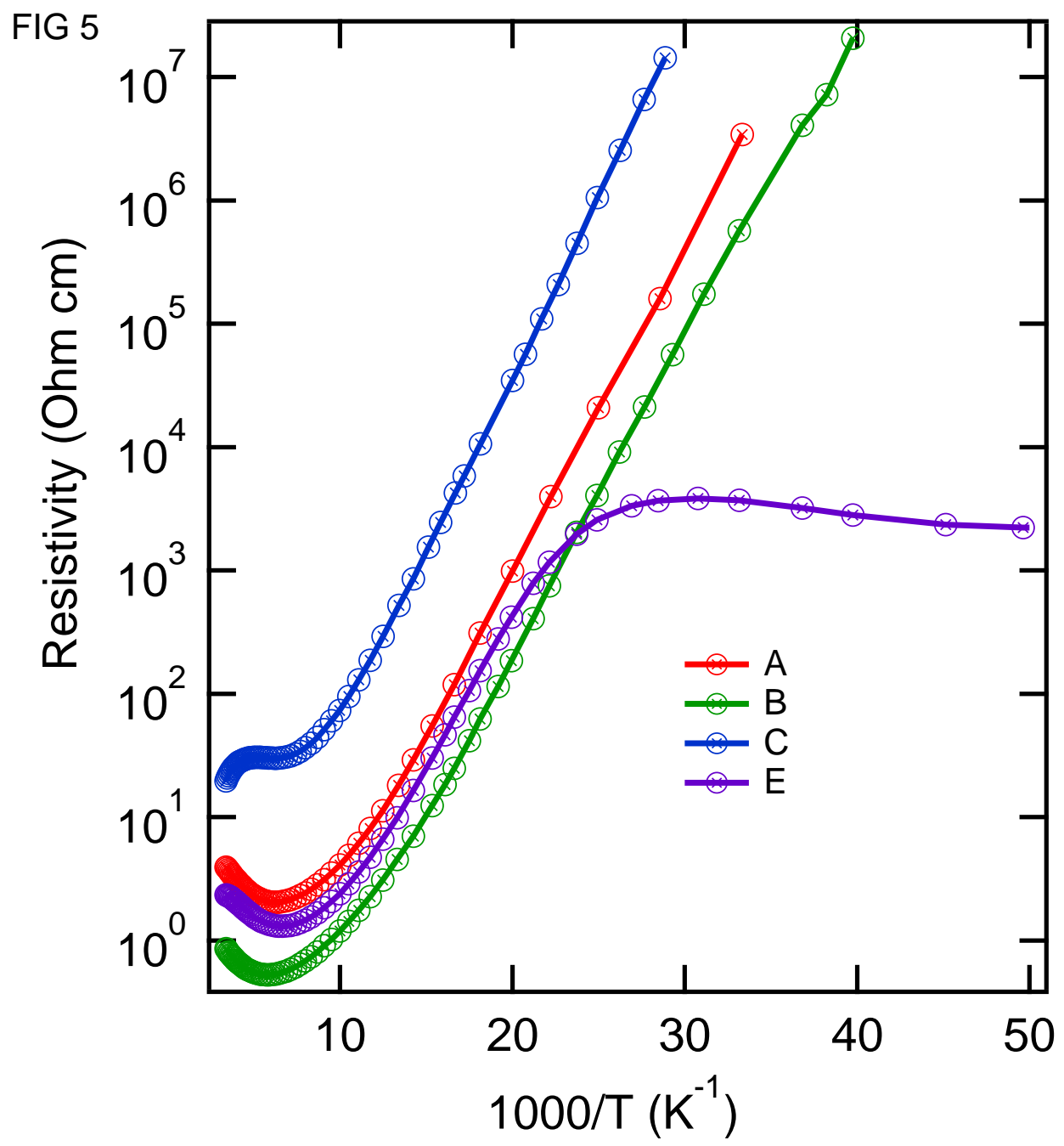

